\title{
Klassische Institutionenökonomik: Kommentar zum Beitrag von Wolfram Elsner
}

\author{
Jan Schnellenbach
}

Online publiziert: 26. Februar 2019

(C) List-Gesellschaft e.V. 2019

\section{Die Perspektive der klassischen Institutionenökonomik}

Wolfram Elsner (2018) hält in seinem Beitrag ein Plädoyer für die Klassische Institutionenökonomik, die nicht mit der in ihren Annahmen letztendlich doch neoklassisch geprägten Neuen Institutionenökonomik zu verwechseln ist (z. B. Richter 2015). In seinem ausführlichen Überblick über die Klassische Institutionenökonomik wird allerdings auch deutlich, dass diese von der methodologischen Seite her äußerst heterogen und - jedenfalls für den konventionell geschulten Ökonomen erst einmal schwer greifbar ist.

So wird beispielsweise als ein wesentliches Merkmal der Fokus auf Historizität und zeit-räumliche Spezifizität gelegt, und es wird betont, dass menschlichem Handeln vielerlei Motive zugrunde lägen. Leicht kann man sich als Leser hier einen Gegensatz zur Mainstream-Ökonomik denken, der, wie von vielen Kritikern immer wieder betont wird, diese Charakteristika angeblich fehlen. Der Blick auf besondere institutionelle Konstellationen, in die ökonomisches Handeln eingebettet ist, wird demnach von Standardökonomen ebenso verweigert wie ein realistisches Menschenbild, das über den angeblich eindimensional gewinn- und nutzenmaximierenden homo oeconomicus hinaus ginge (Hodgson 1989).

Ebenso wird der methodologische Individualismus als wichtige forschungsleitende Annahme der konventionellen Ökonomik stark kritisiert. Stattdessen werden die soziale Einbettung der Individuen und die Interdependenz zwischen ihnen betont. Es wird ein methodologischer Systemismus, oder auch methodologischer Holismus,

This commentary article refers to the article available online at https://doi.org/10.1007/s41025-01900122-x. An author's reply to this commentary article is available online at https://doi.org/10.1007/ s41025-019-00153-4. 
vorgeschlagen, der die sozialen Strukturen, zu denen bei weitem nicht nur formale Institutionen gehören, als Ausgangspunkt der Analyse nimmt.

Zusätzlich bemüht sich die Klassische Institutionenökonomik, auch die naturalistischen Grundlagen menschlichen Handelns zu berücksichtigen: Instinkte, natürliche Bedürfnisse und andere Verhaltensdispositionen, die mehr oder weniger fest verdrahtet sind, werden in ihrer Bedeutung für wirtschaftliche Entscheidungen untersucht. Hinzu kommt dann auch noch eine prozesshafte Perspektive, die berücksichtigen will, dass wirtschaftliche und gesellschaftliche Entwicklungen pfadabhängig und komplex sind. Man wird daher selten konkrete Ereignisse - wie etwa das Auftauchen einer bestimmten Innovation - präzise vorhersagen können. Aber man kann möglicherweise erklären, unter welchen Umständen es zu mehr oder weniger Innovationen kommt und vor allem auch wie eine konkrete Innovation zukünftige wirtschaftliche Entwicklungspfade beeinflusst, bis hin zu ihren Effekten auf die Institutionen. Hier besteht ein Anknüpfungspunkt an Friedrich von Hayeks Argument, dass es in der Ökonomik vor allem um Mustervorhersagen gehe (Hayek 1967).

Damit spielen Institutionen aber in der klassischen Institutionenökonomik eine doppelte Rolle. Einerseits helfen sie zu erklären, wie Menschen entscheiden und miteinander agieren werden. Andererseits aber sind sie selbst als Resultat menschlichen Handelns zu erklären. Nun könnte man einwenden, dass auch die neoklassisch geprägte Neue Institutionenökonomik dies auch tut. Die Theorie der Eigentumsrechte will beispielsweise erklären, wieso es bestimmte Ausprägungen von Eigentumsrechten in der realen Welt gibt, wieso es also aus Sicht von handelnden Individuen rational ist, Eigentumsrechten in unterschiedlichen Abgrenzungen zu definieren. Aber diese Erklärung kann die Theorie nur liefern, wenn sie zuvor versteht, welche Effekte diese Institutionen haben. Der Unterschied zur Klassischen Institutionenökonomik ist nun aber, dass diese explizit von einer zirkulären Kausalität ausgeht. Institutionen beeinflussen das Handeln der Individuen, deren Handeln wiederum die Entwicklung von Institutionen beeinflusst. Damit steht, zumindest in manchen Teilgebieten, der permanente Wandel dem neoklassischen Verständnis von Institutionen als Gleichgewichtsphänomenen gegenüber.

Die Klassische Institutionenökonomik unterscheidet sich also in ihren Annahmen fundamental von der neoklassischen Ökonomik. Unterschiede im Erkenntnisinteresse gibt es dagegen vor allem zur ,traditionellen“ theoretischen Neoklassik, die jedoch seit einiger Zeit selbst im Wandel ist. Dennoch versteht sich die Klassische Institutionenökonomik keinesfalls als bloße Ergänzung zur Neoklassik, die dort anzuwenden wäre, wo die Neoklassik gerade aufgrund ihrer Fokussierung auf Gleichgewichte nicht weiterkommt. Vielmehr sehen die Vertreter der Klassischen Institutionenökonomik ihre Schule als echte Alternative, die auf das gesamte Marktgeschehen und auch auf andere soziale Phänomene anwendbar ist.

\section{Das Modells individuellen Handelns}

Liest man die Ausführungen von Wolfram Elsner in diesem Band, so wird deutlich, dass ein wesentlicher Unterschied zwischen Klassischer Institutionenökonomik und Neoklassik im Modell individuellen Handelns zu finden ist. Die Klassische Institu- 
tionenökonomik strebt demnach ein realistisches Verhaltensmodell an in dem Sinne, dass eine Vielzahl möglicher Einflussgrößen auf menschliches Handeln berücksichtigt werden soll. Die Annahme der Maximierung unter Nebenbedingungen wird als zu stark vereinfachend, ja als geradezu mechanistisch zurückgewiesen. Nun kann man sich allerdings die Frage stellen, was mit einem bis ins Detail realistischen Verhaltensmodell zu gewinnen ist.

Ein erster Einwand kann darin bestehen, dass auch die Mainstream-Ökonomik nicht schläft. Die Erfolgsgeschichte der Verhaltensökonomik hat dazu geführt, dass längst auch eine ganze Reihe psychologischer Einflüsse berücksichtigt werden. Dies geschieht etwa im Rahmen des heuristics and biases-Forschungsprogramms (ursprünglich Tversky und Kahneman 1974), das systematische Abweichungen von vollständiger Rationalität untersucht und das längst ein zentraler Teil des Mainstreams geworden ist. Zwar kann man solchen Ansätzen vorwerfen, dass sie immer noch den hergebrachten homo oeconomicus im engeren Sinne als Maßstab nehmen und teils, etwa im Forschungsprogramm des sogenannten libertären Paternalismus, auch als normatives Ziel wirtschaftspolitischer Eingriffe (Schnellenbach 2014). Aber dennoch: Der Aufstieg der Verhaltensökonomik zeigt, dass auch im Mainstream verankerte Ökonomen bereit sind, das traditionelle ökonomische Verhaltensmodell punktuell anzupassen, wenn empirische Evidenz dies nahelegt.

Ein zweiter und aus meiner Sicht wichtigerer Einwand ist, dass der von der klassischen Institutionenökonomik versprochene umfassende Realismus in den Annahmen die ökonomische Forschung vermutlich nicht wirklich weiterbringen wird. Wie Kirchgässner (2013) ausführlich darlegt, dient das ökonomische Verhaltensmodell primär der Disziplinierung der ökonomischen Analyse. Die Aufgabe der Ökonomen ist es demnach, zu analysieren, wie Menschen systematisch und daher vorhersagbar auf Veränderungen von Randbedingungen ihrer Entscheidungssituationen reagieren. Es ist deshalb überhaupt nicht der Anspruch von Ökonomen, ein ganzheitliches, realistisches Modell allen menschlichen Handelns zu konstruieren. Vielmehr ist es, wenn man Kirchgässner folgt, gerade die Selbstbeschränkung auf die Analyse des individuell rationalen Reagierens auf veränderliche Restriktionen, die ursächlich für den Erfolg der Ökonomik als Sozialwissenschaft in den vergangenen Jahrzehnten ist.

Diese Selbstbeschränkung der Ökonomen kann auch als Teil einer Spezialisierungsstrategie verschiedener akademischer Disziplinen verstanden werden. Dann wäre es beispielsweise die Aufgabe der Soziologie, den homo sociologicus zu analysieren als den Menschen, der sozialen Normen und sonstigen Erwartungen an seine soziale Rolle zu entsprechen sucht (Dahrendorf 1958) und die Aufgabe der Psychologie, beispielsweise reale Entscheidungsprozesse im Detail oder die Herausbildung von Präferenzen zu verstehen. Natürlich lässt sich eine solche disziplinäre Arbeitsteilung nicht vollständig durchhalten; wie wichtig interdisziplinäre Anregungen sein können, zeigt nicht zuletzt die Verhaltensökonomik. Im interdisziplinären Austausch erweist sich allerdings auch das ökonomische Verhaltensmodell als exportfähig. So findet bereits seit längerer Zeit auch in der Soziologie ein Rückgriff auf rational choice-Modelle statt (Hechter und Kanazawa 1997), während dieser Forschungsstrang in der internationalen Politikwissenschaft ohnehin eine längere Tradition hat. Angesichts der zunehmenden Bedeutung des rational choice-Ansatzes in den Sozi- 
alwissenschaften stellt sich daher die Frage, ob ausgerechnet die Ökonomik etwas zu gewinnen hat, wenn sie den homo oeconomicus aufgibt, anstatt ihn vorsichtig zu modifizieren.

Eine solches vorsichtigeres Vorgehen ist möglich, denn auch homo oeconomicus lässt sich je nach konkreter Forschungsfrage flexibel mit Leben füllen. Methodologischer Individualismus und die Annahme des rationalen Handelns sind schließlich zunächst einmal nur formale, forschungsleitende Annahmen. Welche substantiellen Annahmen man dann für die individuelle Präferenzordnung trifft, bleibt dagegen offen. So ist es auch nicht überraschend, dass man bereits relativ früh Modelle findet, in denen interdependente, altruistische Präferenzordnungen unterstellt werden (z.B. Hochman und Rodgers 1969), oder auch solche, die Neid berücksichtigen, oder solche, in denen Individuen aus sich selbst heraus motiviert sein können, eine Leistung zu erbringen (Frey 1997). Nicht zuletzt kann man auch regelbasiertes Verhalten ein Ergebnis individueller Rationalität sein (Heiner 1983). Das ökonomische Verhaltensmodell erweist sich also als erstaunlich flexibel. Dass es kostspielig ist, gegen informale Institutionen zu verstoßen, wenn es einen gewissen Anteil von Individuen gibt, die Regelverletzungen bestrafen, lässt sich ebenso im Standardmodell berücksichtigen. Was gewinnt man also tatsächlich, wenn man das ökonomische Verhaltensmodell aufgibt?

Eine Antwort könnte sein, dass die Klassische Institutionenökonomik als Nachfolgerin der Historischen Schule im jeweiligen Einzelfall genauer hinschaut: Was sind die formalen und informalen Institutionen, die in einer bestimmten historischen Situation gerade einen Einfluss auf das Handeln der Individuen haben? Wie sind wir bei diesen Institutionen gelandet, wieso ist also ein konkreter Markt zu einem bestimmten Zeitpunkt in ein spezifisches Regelsystem eingebettet? Allerdings sollte man nicht vergessen, dass auch die Beantwortung solcher Fragen letztlich eine Reihe von Annahmen über individuelles Verhalten voraussetzt. Wollen wir verstehen, wieso zu einem bestimmten Zeitpunkt sich Individuen mehrheitlich an bestimmten Institutionen orientieren, so müssen wir fragen, wieso es gerade vernünftig ist, dies zu tun - und landen damit wieder beim rational choice-Ansatz. Der Unterschied zwischen einer Klassischen Institutionenökonomik, die einen methodologischen Holismus betreibt, und einer Neoklassik, die Institutionen als Restriktionen individueller Entscheidungen modelliert, verschwimmt dann aber.

Eine konsequente Ablehnung des homo oeconomicus durch die Klassische Institutionenökonomik ist außerdem möglicherweise gerade deshalb problematisch, weil die oben angesprochene Selbstdisziplinierung durch einen harten Kern forschungsleitender Annahmen verloren geht. Liest man den Beitrag von Wolfram Elsner kritisch, so wird dies durchaus deutlich. Man findet eine lange Liste dessen, was die Klassische Institutionenökonomik will, d.h. was sie alles erklären will und was sie alles bereits auf der Ebene ihrer Grundannahmen berücksichtigen möchte. Den Leser lässt dies aber zunächst einmal orientierungslos zurück; die Klassische Institutionenökonomik scheint fast durch ein anything goes gekennzeichnet zu sein. Gerade bei einem methodologischen Holismus scheint die Gefahr zu bestehen, dass theoretische Erklärungen durch ad hoc getroffene Annahmen passend gemacht werden, aber möglicherweise tatsächlich keinen besonders hohen Erklärungsgehalt haben. 


\section{Der empirical turn der Ökonomik}

Besucht man heutzutage eine Fachtagung, oder liest man ein hochrangiges Journal, so fällt auf, dass die Ökonomik zweifellos eine stark empirische Wissenschaft geworden ist und wohl auf absehbare Zeit bleiben wird. Natürlich handelt es sich hier aus Sicht der heterodoxen Kritiker immer noch um eine beklagenswert „enge“ Empirie, in der die ökonometrische Analyse von Daten im Mittelpunkt steht, während andere, etwa narrative Ansätze keine wesentliche Rolle spielen. Aber es ist jedenfalls unumstritten, dass die reine Theorie in der Ökonomik zunehmend marginalisiert ist (Angrist et al. 2017). Viel von der Kritik am Mainstream, die auch die Klassische Institutionenökonomik lange als konstitutives Element pflegte, erscheint damit aber zunehmend obsolet. Wo findet man heutzutage in gut veröffentlichten Papieren noch den ganz eng definierten Rationalclown, der vollständig informiert ist, von keinen formalen und informalen Institutionen geleitet wird und stets für ihn selbst perfekte Entscheidungen trifft?

Im Gegenteil scheint es so zu sein, dass - ohne dies explizit zu wollen - im Mainstream auch Anliegen der klassischen Institutionenökonomik empirisch zunehmend berücksichtigt werden. Man denke etwa an die neue, quantitative Forschungsrichtung in der Wirtschaftsgeschichte, die sich mit den langfristigen Effekten von Institutionen beschäftigt (z. B. Acemoglu et al. 2014), oder auch mit den Auswirkungen, die bestimmte Verhaltensweisen auf unsere Präferenzen und Einstellungen und damit unser wirtschaftliches Handeln in der langen Frist haben (z. B. Bursztyn und Cantoni 2016). Solche Ansätze sind methoden- und datengetrieben. Es gibt sie, weil die Ökonometrie in den letzten Jahrzehnten neue Methoden zur kausalen Inferenz bereitgestellt hat, und weil diese Methoden eine Art Goldgräberstimmung bei der Suche nach zu ihnen passenden Datensätzen ausgelöst haben. Fragestellungen, für die Institutionen und Änderungen von Institutionen bedeutsam sind, eignen sich oft besonders gut für solche Analysen.

Nun könnte man prima facie einwenden, dass die starke empirische Fokussierung der Institutionenforschung im Mainstream mit einer noch nicht adäquaten theoretischen Fundierung einhergeht. Diese Kritik übersieht jedoch, dass entsprechende Papiere in der Regel eben doch auch (mehr oder weniger neoklassisch geprägte) theoretische Hypothesen formulieren. Aber vor allem stellt sich die Frage: Hat denn die Klassische Institutionenökonomik auf der theoretischen Seite mehr zu bieten? Als durchaus wohlwollender Beobachter kann man daran bisher Zweifel haben.

$\mathrm{Zu}$ oft bewegen sich Vertreter der Klassischen Institutionenökonomik immer noch vor allem auf der konzeptionellen Ebene. Sie kritisieren, was der angebliche Mainstream falsch macht und überlegen, was man besser machen sollte. Die Forschungsfragen, zu denen die Klassische Institutionenökonomik überzeugendere theoretische Erklärungen liefert, scheinen dagegen immer noch rar zu sein. Auch Wolfram Elsners Beitrag spiegelt dies wider, denn er bleibt doch relativ unkonkret, wenn es darum geht, anhand spezifischer Forschungsfragen zu demonstrieren, was die Klassische Institutionenökonomik tatsächlich „besser“" macht als etablierte Ansätze.

Für einen Forschungsstrang, der auf eine mehr als 120jährige Tradition zurückblickt, erscheint das wenig überzeugend. Die Klassische Institutionenökonomik hat Verdienste dort, wo sie die Ökonomen auf Forschungsgegenstände hinweist, die sie 
sonst vielleicht übersehen hätten: Wieso gibt es institutionelle Vielfalt, und wieso bringen oft ganz unterschiedliche Institutionen ähnliche ökonomische Resultate hervor? Wieso sind gelegentlich auch ökonomisch ineffiziente Institutionen stabil? Was können wir auf der Makroebene erwarten, wenn Individuen in ihrem Handeln auch durch Emotionen und andere Motive angetrieben sind, die im engen ökonomischen Verhaltensmodell oft übersehen werden? Und so weiter. Auf der methodischen Ebene dagegen gewinnt man den Eindruck, dass die Klassische Institutionenökonomik als Alternative zu den Standardansätzen immer noch nicht viel anzubieten hat.

Dies gilt umso mehr nach dem empirical turn, mit dem die Ökonomik mehr oder weniger das Spielfeld gewechselt hat und damit einen großen Teil der methodischen Kritik der Klassischen Institutionenökonomik am Mainstream veraltet und wenig treffsicher aussehen lässt. Die Tatsache, dass die moderne empirische Ökonomik mit fortgeschrittenen quantitativen Ansätzen Fragestellungen auch aus dem Bereich der Klassischen Institutionenökonomik überzeugender analysiert als die Klassische Institutionenökonomik selbst, lässt jedenfalls an deren zukünftiger Bedeutung Zweifel aufkommen.

\section{Ist die Klassische Institutionenökonomik hilfreich für die ökonomische Politikberatung?}

Man könnte prima facie erwarten, dass eine institutionenökonomische Forschungsrichtung auch in ihren Politikimplikationen sehr institutionenorientiert argumentieren würde. Die Frage könnte sein, welche ökonomischen Auswirkungen unterschiedliche formale Institutionen haben, welche Wechselwirkungen zwischen verschiedenen Institutionen zu bedenken sind und welche Institutionen schließlich aus Sicht der betroffenen Bürger akzeptabel sein könnten. In einem solchen Falle wären die Politikimplikationen nah an einer modernen Ordnungsökonomik (Vanberg 2005), die das gesellschaftsvertragstheoretische Argument der Virginia School um James M. Buchanan aufgreift und vom jeweiligen Status Quo ausgehend fragt, welche institutionellen Reformen nahezu konsensfähig sein könnten. Gerade diesen Weg geht die Klassische Institutionenökonomik aber offenbar nicht.

Wolfram Elsner beschreibt stattdessen das Szenario einer negotiated economy, in der auch Löhne und Preise nicht etwa über den Preismechanismus determiniert werden, sondern mit dem Ziel verhandelt werden, „faire Makrostrukturen“ zu erreichen - ohne genau zu definieren, wann Fairness als gegeben angesehen werden kann. Dies ist vermutlich auch substantiell gar nicht möglich, aber der Ansatz setzt doch voraus, dass eine Arena geschaffen wird, in der Akteure mit unterschiedlichen Interessen in Verhandlungen eintreten können.

Entsprechend zeigen Vertreter der Klassischen Institutionenökonomik oft eine Affinität zu einer korporatistischen Wirtschaftspolitik, die zugleich diskretionär ist. Auf der einen Seite werden Macht- und Hierarchiestrukturen auf Märkten thematisiert, auf der anderen Seite wird oft relativ unkritisch angenommen, dass etwa Gewerkschaften und Arbeitgeberverbände zuverlässige Vertreter der in ihnen gesammelten, oft als relativ homogen angenommenen individuellen Interessen seien. An politischökonomischen Komplikationen ist die Klassische Institutionenökonomik nicht so 
stark interessiert, wie es ihre wirtschaftspolitische Stoßrichtung eigentlich notwendig erscheinen lässt. Probleme wie rent-seeking, das korporatistische Interessenverbände leicht auf Kosten Außenstehender betreiben können, werden beispielsweise allenfalls am Rande behandelt.

Diese Asymmetrie ist durchaus erstaunlich. Macht auf Märkten, die, so sie überhaupt vorhanden ist, in der Regel temporär und über Wettbewerb bestreitbar ist, wird von der Klassischen Institutionenökonomik deutlich kritischer gesehen als politische Macht, in der man im Gegenteil eher ein Heilmittel gegen als problematisch erkannte wirtschaftliche Macht sieht. Dabei spiegelt sich der methodologische Holismus in den wirtschaftspolitischen Ansätzen. Das ökonomische System insgesamt wird als politisch steuerbar angesehen; „faire“ Preis- und Lohnstrukturen können erst von den Beteiligten verhandelt und dann auch wirtschaftspolitisch durchgesetzt werden. Dabei wird Wohlfahrtsverlusten, die durch Verzerrungen markträumender Preise verursacht werden, wenig Aufmerksamkeit geschenkt. Angesichts der andernorts zu findenden Betonung von Komplexität und Innovation erscheint auch der starke Fokus auf wirtschaftspolitische Steuerung und Regulierung als inkonsistent mit anderen Teilen der Klassischen Institutionenökonomik, jedenfalls aber als nicht als geradlinig aus der positiven Theorie folgend.

\section{Schlussfolgerungen}

Es ist das Verdienst der Klassischen Institutionenökonomik, wichtige Fragestellungen auf die Agenda der ökonomischen Forschung gesetzt zu haben und die Beschäftigung mit diesen Fragestellungen immer wieder eingefordert zu haben. Immerhin hat die prozessuale, gar evolutorische Perspektive auf ökonomisches Handeln im neoklassischen Mainstream tatsächlich über Jahrzehnte kaum Beachtung gefunden. Die betreffenden Themen reichen von der Analyse des endogenen Wandels von Institutionen bis hin zu Teilgebieten der Innovationsökonomik. Spätestens nach dem empirical turn gibt es den Mainstream, an dem sich die Klassischen Institutionalisten lange auch als Feindbild abgearbeitet haben, jedoch so nicht mehr. Vielmehr hat der Mainstream es geschafft, auch Themen, die früher heterodoxen Schulen vorbehalten waren, in sein Forschungsprogramm zu integrieren - und dies betrifft beileibe nicht nur die Klassische Institutionenökonomik.

Gleichzeitig ist heute immer weniger klar, was die methodischen Alleinstellungsmerkmale vieler heterodoxer Ansätze sind. Wolfram Elsner zeigt in seinem Aufsatz selbst, wie wichtig beispielsweise die Spieltheorie für die Klassische Institutionenökonomik ist. Sicherlich kommen zwar andere Methoden dazu, die aus der Perspektive des Mainstreams ungewöhnlicher sind, wie etwa Komplexitätstheorie oder agentenbasierte Simulationsmodelle. Hier wird man andererseits aber auch zugeben müssen, dass die großen und überzeugenden Durchbrüche bisher fehlen. Dass es diese Ansätze bisher kaum in die Lehrbücher geschafft haben, liegt eben nicht nur an der Sturheit des Mainstreams, sondern auch daran, dass der Mehrwert einer überzeugenderen Erklärung ökonomischer Phänomene bisher nur sehr selten nachgewiesen wurde. 
Damit stellt sich aber die Frage, ob es überhaupt sinnvoll ist, die Klassische Institutionenökonomik weiterhin als eigenständige, heterodoxe Denkschule zu positionieren. Um sich hierzu ein Urteil zu bilden, müsste die Frage beantwortet werden, ob es sich hier tatsächlich noch um mehr handelt als um ein Buffet sehr heterogener Ansätze, deren gemeinsamer Nenner vor allem ist, nicht Mainstream zu sein - und tendenziell marktskeptische Empfehlungen für die Wirtschaftspolitik zu geben.

\section{Literatur}

Acemoglu, D., Gallego, F. A., \& Robinson, J. A. (2014). Institutions, human capital and development. Annual Review of Economics, 6, 875-912.

Angrist, J., Azoulay, P., Ellison, G., Hill, R., \& Feng, S.L. (2017). Economic research evolves: fields and styles. American Economic Review (P\&P), 107, 293-297.

Bursztyn, L., \& Cantoni, D. (2016). A tear in the iron curtain: the impact of western television on consumption behavior. Review of Economics and Statistics, 91, 25-41.

Dahrendorf, R. (1958). Homo Sociologicus: Versuch zur Geschichte, Bedeutung und Kritik der Kategorie der sozialen Rolle. Kölner Zeitschrift für Soziologie und Sozialpsychologie, 10, 178-208.

Elsner, W. (2018). Klassische Institutionenökonomik. Der „Amerikanische Institutionalismus“, die „Original Institutional Economics“ oder Evolutionär-institutionelle Ökonomik. List-Forum für Wirtschaftsund Finanzpolitik. https://doi.org/10.1007/s41025-019-00122-x

Frey, B. S. (1997). Not Just for the money. An economic theory of personal motivation. Cheltenham: Elgar. von Hayek, F. A. (1967). The theory of complex phenomena. In F. A. von Hayek (Hrsg.), Studies in politics, philosophy and economics (S. 22-42). Chicago: University of Chicago Press.

Hechter, M., \& Kanazawa, S. (1997). Sociological rational choice theory. Annual Review of Sociology, 23, $191-214$.

Heiner, R. (1983). The origins of predictable behavior. American Economic Review, 73, 560-595.

Hochman, H., \& Rodgers, J. (1969). Pareto optimal redistribution. American Economic Review, 59, $542-557$.

Hodgson, G. M. (1989). Institutional economic theory: the old vs. the new. Review of Radical Political Economy, 1(3), 249-269.

Kirchgässner, G. (2013). Homo Oeconomicus. Das ökonomische Modell individuellen Verhaltens und seine Anwendung in den Wirtschafts- und Sozialwissenschaften (4. Aufl.). Tübingen: Mohr Siebeck.

Richter, R. (2015). Essays on new institutional economics. Heidelberg: Springer.

Schnellenbach, J. (2014). Neuer Paternalismus und individuelle Rationalität: eine ordnungsökonomische Analyse. List-Forum für Wirtschafts- und Finanzpolitik, 40, 239-257.

Tversky, A., \& Kahneman, D. (1974). Judgment under uncertainty: heuristics and biases. Science, 185, 1124-1131.

Vanberg, V. J. (2005). Market and State: The Perspective of Constitutional Political Economy. Journal of Institutional Economics, 1, 23-49. 